\title{
Role of MRI in Differentiation between Postoperative Tumoral Recurrence and Radiation-Induced Brain Necrosis in Patients of Glioblastoma Multiform
}

\author{
Jama A. Mohamud ${ }^{1}$, Jianping Gu${ }^{1}$, Salad A. Halane², Farah A. Mohamud ${ }^{3}$ \\ ${ }^{1}$ Department of Radiology, Nanjing First Hospital, Nanjing Medical University, Nanjing, China \\ ${ }^{2}$ Department of Infectious Diseases, Tongji Hospital, Huazhong University of Science and Technology, Wuhan, China \\ ${ }^{3}$ Department of General Surgery, Subei People’s Hospital of Jiangsu Province, Yangzhou University, Yangzhou, China \\ Email: *Anhaar114@hotmail.com
}

How to cite this paper: Mohamud, J.A., $\mathrm{Gu}$, J.P., Halane, S.A. and Mohamud, F.A. (2021) Role of MRI in Differentiation between Postoperative Tumoral Recurrence and Radiation-Induced Brain Necrosis in Patients of Glioblastoma Multiform. Open Journal of Radiology, 11, 45-53.

https://doi.org/10.4236/ojrad.2021.112005

Received: April 2, 2021

Accepted: June 7, 2021

Published: June 10, 2021

Copyright $\odot 2021$ by author(s) and Scientific Research Publishing Inc. This work is licensed under the Creative Commons Attribution International License (CC BY 4.0).

http://creativecommons.org/licenses/by/4.0/

\begin{abstract}
The distinction of radiation-induced brain necrosis (RBN) and recurrent glioblastoma multiform (rGBM) remains a diagnostic challenge due to their similarly on routine follow-up imaging studies and also their clinical manifestations. Our purpose of this review article is to evaluate the role of advanced MR imaging techniques such as Perfusion-weighted imaging (PWI), Diffusion-weighted imaging (DWI) and Magnetic resonance spectroscopy (MRS) in the differentiation of RBN and rGBM and their complications together with our experience and knowledge gained during our neuroimaging practice.
\end{abstract}

\section{Keywords}

MRI, Radiation, Brain Necrosis, Recurrent, Glioblastoma Multiforme

\section{Introduction}

It is well-known that GBM is one of the deadliest forms and fast-growing primary brain tumors in adults, with a poor prognosis despite maximal treatment, the standard treatment of patients with GBM include surgical resection and postoperative chemoradiotherapy [1] [2]. However, most patients develop relapse process after the first treatment which can be treated with re-resection, systemic treatment with targeted agents or cytotoxic chemotherapy, reirradiation, or radio-surgery [3]. Some previous studies indicate that the therapeutic effect of recurrent tumors is not as sensitive as those of primary tumors and in most cases 
of recurrent tumors may invade brain functional areas and lost thus become unresectable. Currently, there is no effective standard treatment for recurrent GBM [4] [5].

RBN is a serious complication of central nervous system caused by combinatorial therapy of brain tumors. The combinatorial therapy is often used to improve the prognosis and to prolong the survival time of glioma patients, but it causes radiation damage to the CNS, which is the most common side effect in gliomas within two years after treatment [6] [7]. Many radiologists believe that conventional CT or MR scan is not effective tool to differentiate recurrence gliomas from radiation necrosis due to their similar characteristics and clinical manifestations [8]. In this review article, we will focus the usefulness of advanced MRI techniques in the differentiation of treatment-induced necrosis and recurrent glioblastoma multiform.

\section{Discussion}

The Imaging differentiation between radiation-induced brain necrosis from recurrent tumor in treated patients with glioblastoma remains a diagnostic challenge [9]. Early stage radiation-induced brain necrosis has no specificity on CT and conventional MRI, which makes it difficult to differentiate from early stage of tumor recurrence, and this may cause to be misdiagnosed and delayed treatment [10] [11]. When the characteristic manifestations of radionecrosis appear on MRI, the pathological changes are often in serious stage [12]. However, the traditional imaging methods cannot distinguish the changes and progress of brain tumors after treatment, but the new advanced imaging diagnosis method such as Perfusion-weighted imaging (PWI), Diffusion-weighted imaging (DWI) and Magnetic resonance spectroscopy (MRS) play very important role to differentiate radiation-induced brain necrosis from recurrent GBM.

\subsection{Radiation-Induced Brain Necrosis}

Radiation-induced brain necrosis is one of the serious complications of radiotherapy for nervous system tumors [13]. In recent years, with the clinical development of improved fractionation techniques (hyperfractionation and hypofractionation), precise Stereotactic radiosurgery (SRS), conformal radiation therapy, and the clinical application of radiosensitizers and radioprotectants it is very important to delve and understand more comprehensively the imaging and histological changes of the central nervous system in different periods after receiving different fractionated regimens, different volumes and doses of radiation, or after receiving therapeutic radiation of combined treatment regimens [14] [15]. Since the emergence of non-invasive neuroimaging diagnosis the understanding of brain radiation injury has been greatly improved, and has attracted the attention of more and more researchers. Brain radiation injury can be divided into acute radiation injury, early delayed injury and late radiation injury [16] [17]. 


\section{1) Acute radiation brain injury}

Acute radiation injury (occurs hours to 3 weeks) it refers to temporary symptoms during or at the end of radiotherapy [18]. It is a rare clinical entity, mainly is due to the damage of blood-brain barrier and increased permeability, resulting in brain edema, increased intracranial pressure and transient neurological impairment [19]. When the volumes of intracranial tumors or craniofacial tumors contain parts of brain tissues, the acute radiation injury is generally mild when the dose and fractionation method of conventional radiotherapy is used. Acute radiation reactions often occur during whole brain or sub whole brain irradiation with several cytotoxic drugs such as cisplatin, cytarabine, methotrexate, interferon and interleukin-2 [20].

\section{2) Early delayed brain injury}

Early delayed radiation injury is usually a transient and reversible white matter injury within a few weeks or three months after radiotherapy, mainly demyelination of oligodendrocytes with axonal edema. The most common is children with acute lymphoblastic leukemia who received 18 - 24 gy prophylactic whole brain irradiation, especially those less than 3 years old. About half of them developed typical "sleep disorder" after 3 - 8 weeks [21] [22].

\section{3) Late stage radiation-induced brain injury}

The clinical symptoms and signs of late stage radiation brain injury and whether the injury is fatal or not depends on the irradiation site, dose and volume, according to the volume range of therapeutic radiation, late radiation injury can be divided into two categories. a) Localized radiation-induced brain necrosis which is the same as local tumor, accompanied by local nervous tissue abnormalities and increased intracranial pressure, clinical symptoms usually appear in 10 months to several years after radiotherapy, about $70 \%$ of patients have symptoms within 2 years after radiotherapy [23] [24]. b) Diffuse radiation-induced brain necrosis: The most characteristic histological changes of late stage radiation brain injury are eosinophilic cells and cellulose exudation and spreading along the gray white matter junction [7]. On CT studies of early radiation-induced brain injury may show low-density changes involving one or both cerebral hemispheres several months after whole brain irradiation, which indicate that there is diffuse white matter abnormalities [25]. This syndrome is common in children with acute lymphoblastic leukemia who received intrathecal methotrexate together with wholebrain radiotherapy, and can also occur in patients who received whole brain or sub whole brain radiotherapy alone. The symptoms of diffuse white matter injury after radiotherapy and chemotherapy usually appear one year after radiotherapy. The most obvious clinical features of severe diffuse white matter injury are mental symptoms, including personality changes, memory loss, attention loss, and dementia. Children mainly show delayed growth and mental development.

\subsection{Recurrent Glioblastoma}

The recurrence of GBM is mostly local, that is, about $2 / 3$ of the tumor recur- 
rence is within $2 \mathrm{~cm}$ of the edge of the primary tumor. One third of the recurrent GBM is far away from the primary tumor, that is, located in different cerebral lobes, contralateral cerebral hemisphere, or even infratentorial [3]. The genetic relationship between primary tumors and distant recurrent tumors remains largely unknown. Some studies speculate that distant recurrence 5 - 10 years after the first operation is actually a new primary tumor; these tumors grow independently and may be caused by adjuvant radiotherapy or chemotherapy [26] [27]. However, other studies have found sufficient genetic similarity between distant recurrent tumors and primary tumors, suggesting that distant recurrent tumors are distant recurrence of primary tumors rather than independent primary GBM [28].

\section{Differentiation between RBN and Recurrent GBM Using MRI Sequences}

The differential diagnosis of postoperative tumoral recurrence and radiation injury remain a scientific and clinical challenge to many experts with the development of MRI fusion technology this ideal multimodal imaging provide more information in the differential diagnosis of postoperative recurrence and radiation-induced brain injury after glioma treatment.

\section{1) Perfusion-weighted imaging (PWI)}

PWI is a well established MRI application that can reflect the distribution of microcirculation and blood perfusion, and can provide information on hemodynamics of tissue or lesion, the perfusion curve can be used to calculate the relative cerebral blood volume (rCBV), relative cerebral blood flow (rCBF) and mean transit time (MTI') [29]. These indexes can provide semi quantitative hemodynamic parameters and quantitatively describe the structure and function of microvessels in the target area. Currently, the main parameter of PWI for differentiating radiation necrosis from tumor recurrence is CBV due to the lack of neovascularization in typical radionecrosis, vascular endothelial cell necrosis, vascular wall cellulose necrosis and vitreous degeneration, angiogenic edema, vascular lumen stenosis and other changes, hence, as the CBV values decrease the blood supply of the recurrent tumor increases due to a large number of new blood vessels. PWI can show the change of $\mathrm{rCBV}$ in radiation-induced brain injury area, which is related to the severity of injury and radiation dose. However, some studies do not support the above results, sometimes there is no significant difference in blood perfusion between radiation necrosis and recurrent tumor [30] [31]. This could be that the blood-brain barrier (BBB) is damaged by radiation injury and the leakage of contrast medium through $\mathrm{BBB}$ affects the local magnetic field, and affects the measurement of CBF and CBV. In the clinical follow-up process of GBM after radiotherapy, the new abnormal enhancement lesions are often mixed lesions with radiation damage and tumor tissues, but the proportion of different lesions is different, and some lesions remain to be the main target. The degree of tumor neovascularization is not always obvious when 
some low-grade gliomas recur. In addition, tumor recurrence usually occurs after irradiation of brain tissues; therefore, there is still some overlap between radiation injury and tumor recurrence CBV.

GBMs has abundant blood supply and relatively high blood flow, the cerebral blood flow (CBF) can be evaluated by using PWI. The CBF of recurrent GBM is relatively high, the local blood flow perfusion is rich and the tissue activity is high. Sugahara et al. [32] study found that when the rCBV is less than $0.6 \mathrm{ml} / 100 \mathrm{~g}$ can be diagnosed as radiation-induced necrosis, and when the $\mathrm{rCBV}$ is greater than $2.6 \mathrm{ml} / 100 \mathrm{~g}$ can be considered as a tumor recurrence but when the rCBV is between 0.6 and $2.6 \mathrm{ml} / 100 \mathrm{~g}$, it is difficult to determine whether it is tumor progression or imaging changes after treatment, and therefore it will need a further imaging studies to reach an accurate diagnosis.

\section{2) Diffusion-weighted imaging (DWI)}

DWI can capture the diffusion motion of water molecules inside brain volumes that can be used to describe the structure of the tissue at the cellular level by using apparent diffusion coefficient (ADC) [33]. The ADC values in DWI can reflect the density of tumor cells and the changes of tissue structure at the cellular level, the higher the density of tissue cells, the more limited the water molecules movement and the lower the ADC values. There are many clinical studies on DWI in the differentiating postoperative recurrence of GBM from radiation-induced brain injury but their results are different [34]. This could be due to the selection of ROI and some other factors, Because of the complex pathology of the operation area after operation and radiotherapy, some enhanced lesions may have both recurrent tumor components and tissue response to treatment. Gliosis and macrophage infiltration may reduce ADC value, and necrosis and bleeding may also have a greater impact on ADC value. In order to reduce the error, it's better to analyze the ADC value of the whole lesion instead measuring the ROI manually. DWI is a routine scanning sequence in clinic, the ADC value of tumor parenchyma has an important reference value in differentiating postoperative recurrence of glioma from radiation-induced brain injury. However the different measurement of regions of interest has a great impact on the results, which still needs further study.

\section{3) Magnetic resonance spectroscopy (MRS)}

MRS is a noninvasive technique for quantitative detection of metabolites in tissues. Proton magnetic resonance spectroscopy ( ${ }^{1} \mathrm{H}$-MRS) is commonly used in clinic. ${ }^{1} \mathrm{H}$-MRS is able to detect the metabolites such as n-acetylaspartic (NAA), creatine (CR), choline (CHO), lipid (LIP) and lactic acid which enrich the differential thinking of brain tumor recurrence and radiation-induced brain necrosis [35]. The peak of NAA decreases when the nerve cells are destroyed due to the infiltrative growth of tumor cells, the higher degree of malignancy, the lower peak level of the NAA. The increase of CHO complex may be due to the abnormal increase of membrane metabolism of tumor cells. Creatine $(\mathrm{Cr})$ shows high energy pathway in energy metabolism, and its decrease is related to energy dep- 
letion and lack of blood flow due to rapid cell proliferation. The presence of lactic acid (LAC) indicates lack of energy metabolism, and the presence of lipid (LIP) is a sign of necrotic tissues, which cannot be detected in low-grade tumors, while the lipid peak are more obvious in high-grade tumors due to necrosis [36]. Talor et al. [37] found that the levels of NAA, creatine and choline drop off in the area of radiation necrosis, while the levels and peak values of creatine and choline increase moderately in the area of tumor recurrence. Another study done by Zeng et al. [38] were used 3D MRS to evaluate the new enhanced lesions in the original area after glioma treatment, and found that the CHO/NAA and $\mathrm{Cho} / \mathrm{Cr}$ ratios of radiation-induced brain necrosis were lower than those of tumor recurrence.

\section{Conclusion}

Reaching accurate differential diagnosis of glioma recurrence and radiation-induced brain injury after GBM therapy is great important for the choice of treatment and prognosis of patients. The follow-up observation of MRI can help to distinguish the recurrence of tumor and change after treatment. Perfusion and diffusion imaging, magnetic resonance spectroscopy and texture analysis can reflect the information of tissue function and metabolism from many aspects, and can effectively identify from one other.

\section{Conflicts of Interest}

The authors declare no conflicts of interest regarding the publication of this paper.

\section{References}

[1] Mary Elizabeth, D. (2016) Glioblastoma: Overview of Disease and Treatment. Clinical Journal of Oncology Nursing, 20, S2-S8. https://doi.org/10.1188/16.CJON.S1.2-8

[2] Villà, S., Balañà, C. and Comas, S. (2014) Radiation and Concomitant Chemotherapy for Patients with Glioblastoma Multiforme. Chinese Journal of Cancer, 33, 25-31. https://doi.org/10.5732/cjc.013.10216

[3] Roy, S., Lahiri, D., Maji, T. and Biswas, J. (2015) Recurrent Glioblastoma: Where We Stand. South Asian Journal of Cancer, 4, 163-173. https://doi.org/10.4103/2278-330X.175953

[4] Young, R.M., Jamshidi, A., Davis, G. and Sherman, J.H. (2015) Current Trends in the Surgical Management and Treatment of Adult Glioblastoma. Annals of Translational Medicine, 3, Article No. 121.

[5] Rees, J.H. (2011) Diagnosis and Treatment in Neuro-Oncology: An Oncological Perspective. British Journal of Radiology, 84, S82-S89. https://doi.org/10.1259/bjr/18061999

[6] Walker, A.J., Ruzevick, J., Malayeri, A.A., Rigamonti, D., Lim, M., Redmond, K.J., et al. (2014) Postradiation Imaging Changes in the CNS: How Can We Differentiate between Treatment Effect and Disease Progression? Future Oncology, 10, 1277-1297. https://doi.org/10.2217/fon.13.271 
[7] Vellayappan, B., Tan, C.L., Yong, C., Khor, L.K., Koh, W.Y., Yeo, T.T., et al. (2018) Diagnosis and Management of Radiation Necrosis in Patients with Brain Metastases. Frontiers in Oncology, 8, Article No. 395. https://doi.org/10.3389/fonc.2018.00395

[8] Verma, N., Cowperthwaite, M.C., Burnett, M.G. and Markey, M.K. (2013) Differentiating Tumor Recurrence from Treatment Necrosis: A Review of Neuro-Oncologic Imaging Strategies. Neuro-Oncology, 15, 515-534. https://doi.org/10.1093/neuonc/nos307

[9] Nael, K., Bauer, A.H., Hormigo, A., Lemole, M., Germano, I.M., Puig, J. and Stea, B. (2018) Multiparametric MRI for Differentiation of Radiation Necrosis from Recurrent Tumor in Patients with Treated Glioblastoma. American Journal of Roentgenology, 210, 18-23. https://doi.org/10.2214/AJR.17.18003

[10] Zikou, A., Sioka, C., Alexiou, G.A., Fotopoulos, A., Voulgaris, S. and Argyropoulou, M.I. (2018) Radiation Necrosis, Pseudoprogression, Pseudoresponse, and Tumor Recurrence: Imaging Challenges for the Evaluation of Treated Gliomas. Contrast Media \& Molecular Imaging, 2018, Article ID: 6828396. https://doi.org/10.1155/2018/6828396

[11] Shah, R., Vattoth, S., Jacob, R., Manzil, F.F.P., O’Malley, J.P., Borghei, P., Patel, B.N. and Curé, J.K. (2012) Radiation Necrosis in the Brain: Imaging Features and Differentiation from Tumor Recurrence. Radiation Oncology, 31, 1343-1359. https://doi.org/10.1148/rg.325125002

[12] Chao, S.T., Ahluwalia, M.S., Barnett, G.H., Stevens, G.H.J., Murphy, E.S., Stockham, A.L., Shiue, K. and Suh, J.H. (2013) Challenges with the Diagnosis and Treatment of Cerebral Radiation Necrosis. International Journal of Radiation Oncology, Biology, Physics, 87, 449-457. https://doi.org/10.1016/j.ijrobp.2013.05.015

[13] Miyatake, S., Nonoguchi, N., Furuse, M., Yoritsune, E., Miyata, T., Kawabata, S., et al. (2015) Pathophysiology, Diagnosis, and Treatment of Radiation Necrosis in the Brain. Neurologia Medico-Chirurgica, 55, 50-59. https://doi.org/10.2176/nmc.ra.2014-0188

[14] Kirkpatrick, J.P., Soltys, S.G., Lo, S.S., Beal, K., Shrieve, D.C. and Brown, P.D. (2017) The Radiosurgery Fractionation Quandary: Single Fraction or Hypofractionation? Neuro-Oncology, 19, ii38-ii49. https://doi.org/10.1093/neuonc/now301

[15] Sawamura, Y., Shirato, H., Ikeda, J., Tada, M., Ishii, N., Kato, T., Abe, H. and Fujieda, K. (1998) Induction Chemotherapy Followed by Reduced-Volume Radiation Therapy for Newly Diagnosed Central Nervous System Germinoma. Journal of Neurosurgery, 88, 66-72. https://doi.org/10.3171/jns.1998.88.1.0066

[16] Greene-Schloesser, D., Robbins, M.E., Peiffer, A.M., Shaw, E.G., Wheeler, K.T. and Chan, M.D. (2012) Radiation-Induced Brain Injury: A Review. Frontiers in Oncology, 2, Article No. 73. https://doi.org/10.3389/fonc.2012.00073

[17] Akita, S. (2014) Treatment of Radiation Injury. Advances in Wound Care, 3, 1-11. https://doi.org/10.1089/wound.2012.0403

[18] Turnquist, C., Harris, B.T. and Harris, C.C. (2020) Radiation-Induced Brain Injury: Current Concepts and Therapeutic Strategies Targeting Neuroinflammation. Neuro-Oncology Advances, 2, vdaa057. https://doi.org/10.1093/noajnl/vdaa057

[19] Sorby-Adams, A.J., Marcoionni, A.M., Dempsey, E.R., Woenig, J.A. and Turner, R.J. (2017) The Role of Neurogenic Inflammation in Blood-Brain Barrier Disruption and Development of Cerebral Oedema Following Acute Central Nervous System (CNS) Injury. International Journal of Molecular Sciences, 18, Article No. 1788. https://doi.org/10.3390/ijms18081788 
[20] Lawrence, Y.R., Li, X.A., el Naqa, I., Hahn, C.A., Marks, L.B., Merchant, T.E., et al. (2010) Radiation Dose-Volume Effects in the Brain. International Journal of Radiation Oncology, Biology, Physics, 76, S20-S27. https://doi.org/10.1016/j.ijrobp.2009.02.091

[21] Yang, Z., Bai, S., Gu, B., Peng, S., Liao, W. and Liu, J. (2015) Radiation-Induced Brain Injury after Radiotherapy for Brain Tumor. In: Lichtor, T., Ed., Molecular Considerations and Evolving Surgical Management Issues in the Treatment of Patients with a Brain Tumor, IntechOpen, London. https://doi.org/10.5772/59045

[22] Kessler, A.T. and Bhatt, A.A. (2018) Brain Tumour Post-Treatment Imaging and Treatment-Related Complications. Insights Imaging, 9, 1057-1075. https://doi.org/10.1007/s13244-018-0661-y

[23] Zhuang, H., Shi, S., Yuan, Z. and Chang, J.Y. (2019) Bevacizumab Treatment for Radiation Brain Necrosis: Mechanism, Efficacy and Issues. Molecular Cancer, 18, Article No. 21. https://doi.org/10.1186/s12943-019-0950-1

[24] Spałek, M. (2016) Chronic Radiation-Induced Dermatitis: Challenges and Solutions. Clinical, Cosmetic and Investigational Dermatology, 9, 473-482. https://doi.org/10.2147/CCID.S94320

[25] Castellano, A. and Anzalone, N. (2019) Radiation and Chemotherapy Induced Injury. In: Barkhof, F., Jäger, H., Thurnher, M. and Rovira, À., Eds., Clinical Neuroradiology, Springer, Cham.

[26] Takeshita, T., Yan, L., Asaoka, M., Rashid, O. and Takabe, K. (2019) Late Recurrence of Breast Cancer Is Associated with Pro-Cancerous Immune Microenvironment in the Primary Tumor. Scientific Reports, 9, Article No. 16942.

https://doi.org/10.1038/s41598-019-53482-x

[27] Vilalta, M., Rafat, M. and Graves, E.E. (2016) Effects of Radiation on Metastasis and Tumor Cell Migration. Cellular and Molecular Life Sciences, 73, 2999-3007. https://doi.org/10.1007/s00018-016-2210-5

[28] Kraboth, Z. and Kalman, B. (2020) Longitudinal Characteristics of Glioblastoma in Genome-Wide Studies. Pathology \& Oncology Research, 26, 2035-2047. https://doi.org/10.1007/s12253-019-00705-1

[29] Pauliah, M., Saxena, V., Haris, M., Husain, N., Rathore, R.K.S. and Gupta, R.K. (2007) Improved $T_{1}$-Weighted Dynamic Contrast-Enhanced MRI to Probe Microvascularity and Heterogeneity of Human Glioma. Magnetic Resonance Imaging, 25, 1292-1299. https://doi.org/10.1016/j.mri.2007.03.027

[30] Tekşam, M., Kayahan, E.M., Yerli, H. and Ağildere, A.M. (2004) Radyasyon nekrozu-tümör rekürensi ayriminda MR perfüzyon ve MR spektroskopi [Brain MR Perfusion and MR Spectroscopy in Differentiation of Radiation Necrosis from Tumor Recurrence (Case Report)]. Tani Girisim Radyol, 10, 263-267. (in Turkish)

[31] Chuang, M.T., Liu, Y.S., Tsai, Y.S., Chen, Y.C. and Wang, C.K. (2016) Differentiating Radiation-Induced Necrosis from Recurrent Brain Tumor Using MR Perfusion and Spectroscopy: A Meta-Analysis. PLOS ONE, 11, e0141438.

https://doi.org/10.1371/journal.pone.0141438

[32] Sugahara, T., Korogi, Y., Tomiguchi, S., Shigematsu, Y., Ikushima, I., Kira, T., Liang, L., Ushio, Y. and Takahashi, M. (2000) Posttherapeutic Intraaxial Brain Tumor: The Value of Perfusion-Sensitive Contrast-Enhanced MR Imaging for Differentiating Tumor Recurrence from Nonneoplastic Contrast-Enhancing Tissue. American Journal of Neuroradiology, 21, 901-909.

[33] Padhani, A.R., Koh, D.M. and Collins, D.J. (2011) Whole-Body Diffusion-Weighted MR Imaging in Cancer: Current Status and Research Directions. Radiology, 261, 
700-718. https://doi.org/10.1148/radiol.11110474

[34] Drake-Pérez, M., Boto, J., Fitsiori, A., Lovblad, K. and Isabel Vargas, M. (2018) Clinical Applications of Diffusion Weighted Imaging in Neuroradiology. Insights into Imaging, 9, 535-547. https://doi.org/10.1007/s13244-018-0624-3

[35] Doblas, S., He, T., Saunders, D., Hoyle, J., Smith, N., Pye, Q., et al. (2012) In Vivo Characterization of Several Rodent Glioma Models by ${ }^{1} \mathrm{H}$ MRS. NMR in Biomedicine, 25, 685-694. https://doi.org/10.1002/nbm.1785

[36] Nakamura, H., Doi, M., Suzuki, T., Yoshida, Y., Hoshikawa, M., Uchida, M., et al. (2018) The Significance of Lactate and Lipid Peaks for Predicting Primary Neuroepithelial Tumor Grade with Proton MR Spectroscopy. Magnetic Resonance in Medical Sciences, 17, 238-243. https://doi.org/10.2463/mrms.mp.2017-0042

[37] Zeng, Q.S., Li, C.F., Zhang, K., Liu, H., Kang, X.S. and Zhen, J.H. (2007) Multivoxel 3D Proton MR Spectroscopy in the Distinction of Recurrent Glioma from Radiation Injury. Journal of Neuro-Oncology, 84, 63-69.

https://doi.org/10.1007/s11060-007-9341-3

[38] Taylor, J.S., Langston, J.W., Reddick, W.E., Kingsley, P.B., Ogg, R.J, Pui, M.H., et al. (1996) Clinical Value of Proton Magnetic Resonance Spectroscopy for Differentiating Recurrent or Residual Brain Tumor from Delayed Cerebral Necrosis. International Journal of Radiation Oncology, Biology, Physics, 36, 1251-1261.

https://doi.org/10.1016/S0360-3016(96)00376-8 